\title{
Ligas acadêmicas e o potencial de inclusão: confecção da peça anatômica do olho
}

\author{
Academic leagues and the potential for inclusion: confection the anatomical piece of the eye \\ Las ligas académicas y el potencial de inclusión: confección de la trozo anatómica del ojo
}

Recebido: 10/01/2022 | Revisado: 20/01/2022 | Aceito: 03/02/2022 | Publicado: 05/02/2022

\author{
Bruna Alves Bezerra \\ ORCID: https://orcid.org/0000-0003-4142-964X \\ Faculdade Carajás, Brasil \\ E-mail: bruna14bezerra@gmail.com \\ Lilia de Souza Moura \\ ORCID: https://orcid.org/0000-0002-8696-9466 \\ Faculdade Carajás, Brasil \\ E-mail: liliamoura3@gmail.com \\ Rogério do Espírito Santo Amorim Correa \\ ORCID: https://orcid.org/0000-0001-6066-8406 \\ Universidade do Estado do Pará, Brasil \\ E-mail: amorimcorrea10@gmail.com \\ Lucas Henrique de Amorim Lima \\ ORCID: https://orcid.org/0000-0001-6757-6360 \\ Universidade do Estado do Pará, Brasil \\ E-mail: lucas.alima@aluno.uepa.br \\ Samuel Filipe Lopes Alves \\ ORCID: https://orcid.org/0000-0003-1437-5674 \\ Universidade do Estado do Pará, Brasil \\ E-mail: samuel.alves@aluno.uepa.br \\ Mírian Rosa Pereira \\ ORCID: https://orcid.org/0000-0002-8157-1192 \\ Universidade do Estado do Pará, Brasil \\ E-mail: mirianpereira@uepa.br \\ Cilene Aparecida de Souza Melo \\ ORCID: https://orcid.org/0000-0003-1170-6466 \\ Universidade do Estado do Pará, Brasil \\ Faculdade Carajás, Brasil \\ E-mail: cilene@uepa.br
}

\begin{abstract}
Resumo
Objetivo: produção da peça anatômica de baixo custo do olho para deficientes visuais. Metodologia: Trata-se de um estudo de natureza experimental, qualitativa e descritiva que objetiva a confecção do modelo anatômico do olho para ensino-aprendizagem, sendo confeccionado com materiais de baixo custo com ênfase para pessoas com deficiências visuais. Resultados e discussão: A peça anatômica apresenta nas superfícies de cada pequena estrutura relevos e textura especifica, onde se atentou as diferentes moldagens estruturais para uma sensibilidade tátil diferenciada dos deficientes visuais. Os materiais de baixo custo foram escolhidos em decorrência de sua maior durabilidade, resistência, maleabilidade manual e sob o calor, tempo de secagem, transparência e leveza, além do uso de uma coloração contrastante na peça anatômica, permitindo a estimulação visual do aluno de baixa visão. Considerações finais: Dado o exposto, conclui-se que o modelo tridimensional sendo confeccionado com materiais de baixo custo, apresenta um método alternativo para o ensino aprendizado de educandos com deficiência visual e baixa visão.
\end{abstract}

Palavras-chave: Anatomia educação de pessoas com deficiência visual; Ensino; Modelos anatômicos.

\begin{abstract}
Objective: production of a low-cost anatomical piece of the eye for the visually impaired. Methodology: This is an experimental, qualitative and descriptive study that aims to make an anatomical model of the old teaching-learning school, being made with low-custody materials as a stage for people with visual impairments. Results and discussion: A small anatomical surface appears on the surfaces of each small structure, relays and specific texture, where different structural molds were used for a differentiated tactile sensitivity of two visual impairments. Low-custody materials were chosen due to their greater durability, strength, manual malleability and heat, drying time, transparency and lightness, in addition to the use of a contrasting coloration on the anatomical body, allowing the visual stimulation of the lowlevel student. eyesight. Final Considerations: Given or exposed, it is concluded that the three-dimensional model, being made with low-guard materials, presents an alternative method of learning with students with visual impairment and low vision.
\end{abstract}

Keywords: Anatomy; Education of visually disabled; Teaching; Models, anatomic. 


\begin{abstract}
Resumen
Objetivo: producción de una pieza anatómica del ojo de bajo costo para personas con discapacidad visual. Metodología: Se trata de un estudio experimental, cualitativo y descriptivo que tiene como objetivo realizar un modelo anatómico de la antigua escuela de enseñanza-aprendizaje, siendo elaborado con materiales de baja custodia como escenario para personas con discapacidad visual. Resultados y discusión: Aparece una pequeña superficie anatómica en las superficies de cada pequeña estructura, relés y textura específica, donde se utilizaron diferentes moldes estructurales para una sensibilidad táctil diferenciada de dos deficiencias visuales. Se eligieron materiales de baja custodia por su mayor durabilidad, resistencia, maleabilidad manual y calor, tiempo de secado, transparencia y ligereza, además del uso de una coloración contrastante en el cuerpo anatómico, permitiendo la estimulación visual del alumno de bajo nível del vista. Consideraciones finales: Dado o expuesto, se concluye que el modelo tridimensional, al estar elaborado con materiales de baja guardia, presenta un método alternativo de aprendizaje con estudiantes con discapacidad visual y baja visión.
\end{abstract}

Palabras clave: Anatomía; Educación de personas con discapacidad visual; Enseñanza; Modelos anatómicos.

\title{
1. Introdução
}

A deficiência visual, assim como muitas outras, não era vista pela maioria das sociedades antigas como algo aceitável, e os fortes aspectos culturais que as permeavam eram determinantes para que as pessoas com deficiência fossem de forma natural excluídas dos grupos a que pertenciam (Costa; Picharillo e Paulino, 2018). Porém, com o passar dos séculos, houve uma redução gradual do olhar preconceituoso voltado aos deficientes, sendo o Século das Luzes (XVIII) um importante marco para essa mudança (Fernandes et al., 2011)

Nessa perspectiva, foi principalmente na Europa que os primeiros movimentos pelo atendimento aos deficientes, refletindo mudanças na atitude dos grupos sociais, se concretizaram em medidas educacionais (Rogalski, 2010). O Brasil também manifestou maior preocupação com a educação especial, o que fez com que D. Pedro II promulgasse um decreto imperial em 1854 para a criação do Instituto dos Meninos Cegos para apoio e redução das limitações dos mesmos (atualmente é o Instituto Benjamin Constant) (Leão; Sofiato, 2019). Sobre a educação especial voltada para cegos, alguns elementos são essenciais para que seja efetiva, como a valorização das experiências táteis, auditivas e cinestésicas, ou seja, a colocação do estudante deficiente no centro do processo de aprendizado (Nuernberg, 2008).

Entretanto, não pode ser desconsiderada a existência de disciplinas com alto grau de complexidade e que por isso tornam seu aprendizado por deficientes visuais mais difíceis, como principais exemplos temos as matérias da área morfológica, sendo a anatomia uma delas, pois a impossibilidade de visualizar imagens digitais ou esquemas didáticos são determinantes para tornar o repasse dos conhecimentos dessa área mais dificultoso. Desse modo, ao se considerar os objetivos da Política Nacional de Educação Especial (PNEE) (Brasil, 2020), compreende-se a necessidade de que os estudantes tenham suas especificidades atendidas da forma mais eficiente possível no âmbito educacional, sendo a deficiência visual uma delas.

Nesse sentido, é um fato preocupante a pouca disponibilidade de materiais especializados para a educação especial em colégios e universidades, sendo que quando existem, em sua grande maioria não são adequados para que haja um aprendizado efetivo (Sant'Anna, 2014). Além disso, o alto custo e a aquisição em pouca quantidade desses itens não permitem que sejam oferecidos para uso individual (Garcez, 2014). Tratando com realismo a temática, a elaboração desses recursos direcionados à educação especial deve ser simples, barata e com informações técnicas precisas (Cerqueira \& Ferreira, 2000).

Desse modo, contempla-se a importância deste trabalho, o qual visou a confecção da peça anatômica de baixo custo do olho para deficientes visuais, uma vez que poderá facilitar o ensino da anatomia para esse grupo, algo ainda mais relevante ao se considerar que tal disciplina tende a ser ainda mais complexa para tais deficientes. Nesse contexto, o objetivo desse estudo foi confeccionar peça anatômica de baixo custo que possa contribuir no processo de ensino-aprendizagem de pessoas com deficiência visual. 


\section{Metodologia}

Trata-se de um estudo de natureza experimental, qualitativa e descritiva que objetiva a confecção do modelo anatômico do olho para ensino-aprendizagem. De acordo com Praça (2015), a pesquisa experimental envolve a manipulação de variáveis relacionadas ao objeto de estudo, através da realização de experimentos que auxiliam no desenvolvimento da pesquisa, no caso do presente estudo, o objeto de estudo foi confeccionado com materiais de baixo custo com ênfase para pessoas com deficiências visuais.

A pesquisa será desenvolvida no município de Marabá, localizado no sudeste do estado do Pará que apresenta população estimada em 283.542 habitantes e área territorial de $15.128,058 \mathrm{~km}^{2}$. O projeto é uma iniciativa do departamento de pesquisa da Liga Acadêmica de Anatomia Humana e Clínica - LIANAC e Núcleo de Acessibilidade, Educação e Saúde - NAES da Universidade Estadual do Pará - UEPA - Campus VIII, localizado av. Hiléia, Agrópolis do Incra s/n - Amapá, Marabá - PA em parceria com a Faculdade Carajás (IBGE, 2019).

O planejamento para confecção da peça sucedeu através dos livros/atlas de Anatomia Humana da biblioteca e acervo didático do laboratório de Anatomia da Faculdade Carajás para a busca de modelos específicos. Em seguida, a escolha de materiais de baixo custo com fácil manuseio para construção da peça anatômica (Figura 1).

Para elaboração do modelo didático foram utilizados os seguintes itens:

- Estruturas anatômicas: bola de isopor oco de tamanho médio, massa biscuit de cor branca 900g, bastão de cola, fita adesiva crepe tamanho grande, cola de isopor, estilete, placa de isopor com espessura grossa, papel cartão, tintas, tesoura, régua de $30 \mathrm{~cm}$, caneta de cor preto, papelão, espátula e palito, verniz, pano de algodão, pincéis, garrafa pet e pasta plástica.

Papel machê: papéis recicláveis, cola branca de $500 \mathrm{~g}, 100 \mathrm{ml}$ de hipoclorito de sódio, $100 \mathrm{ml}$ de amaciante, 2,5L de água e dois recipientes de $20 \mathrm{~L}$.

Figura 1. Materiais de baixo custo.

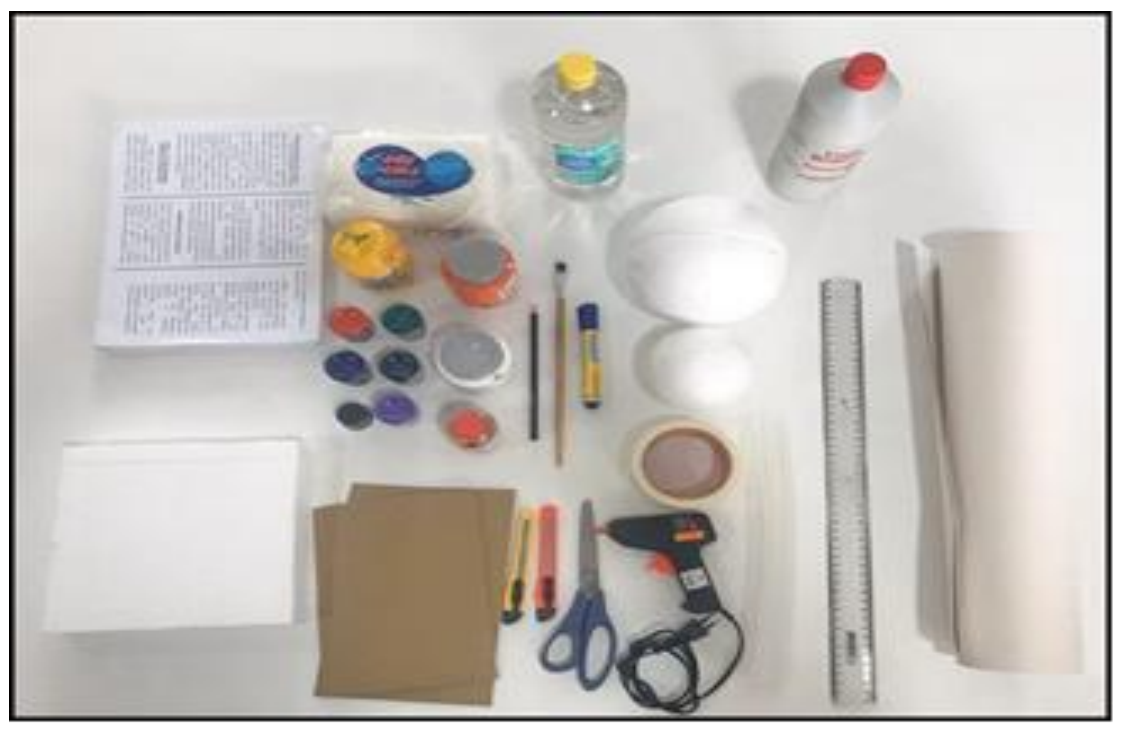

Fonte: Autores.

O papel machê foi a base da confecção do modelo anatômico, por isso para sua composição foi necessário realizar várias etapas até obter uma mistura homogênea e de fácil manuseio, para chegar a tal resultado, utilizou-se a técnica de picagem dos papéis recicláveis e armazenamento em recipientes com misturas líquidas que favorece a umidificação e a trituração dos papéis, seja por fricções manuais ou mecânica (Figura 2). 
Figura 2. Papéis de molho na mistura líquida.

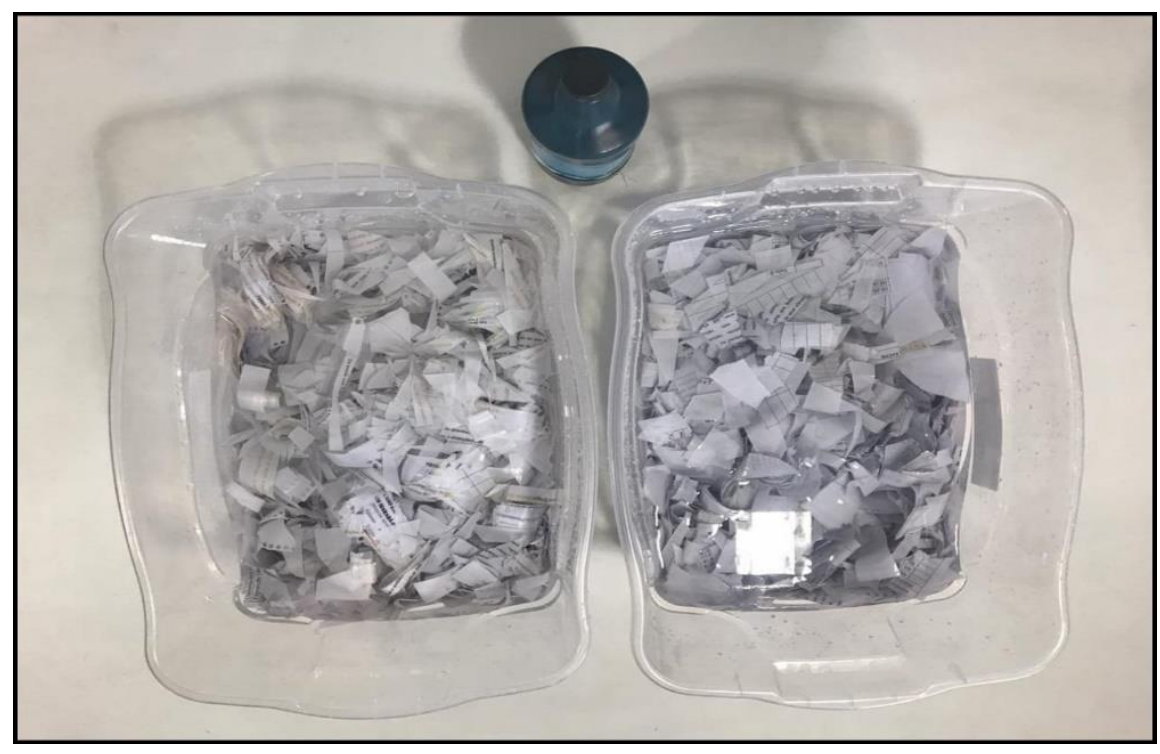

Fonte: Autores.

Em seguida foi moldando aos poucos a peça com papel machê e massa biscuit como representado na figura 3. A esfera de isopor foi revestida com papel machê representando a esclera. Após isso, realizamos a modelagem da retina com massa biscuit e da camada coróide com papel machê. A figura 3 apresenta as divisões da peça anatômica, no qual pode-se observar: Letra A: esclera. Letra B: retina. Letra C: coróide. Letra D: esclera vista posterior do olho. Cada uma dessas estruturas apresenta relevos e texturas diferentes de modo que possam ser identificadas através do toque.

Figura 3. Divisões da peça anatômica.
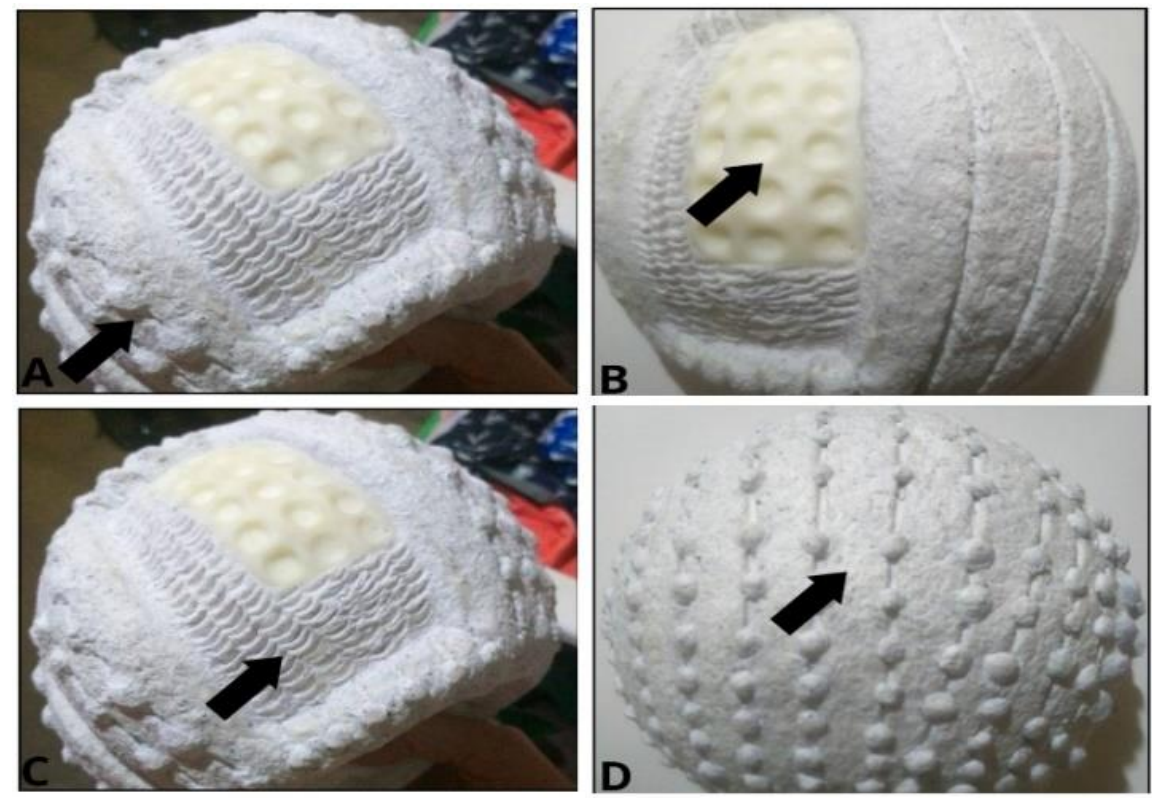

Fonte: Autores.

Deu-se continuidade na confecção da peça através da representação da córnea por meio de uma capa plástica transparente de caderno, em seguida elaborou-se o cristalino, no qual utilizou-se garrafa pet para a confecção da estrutura, buscando o mais realístico possível. Em sequência a íris, músculos e nervo óptico, veias, artérias, fóvea e corpo ciliar, sendo 
essas últimas estruturas citadas confeccionada por massa biscuit. Por fim, foi moldado o humor vítreo através de cola quente, pensando sempre pela maior originalidade da parte estrutural da peça. O processo de confecção do modelo anatômico finalizou com a pintura detalhada e com cores variadas para diferenciar cada estrutura, visto que cores vibrantes podem auxiliar na identificação de pessoas com baixa visão.

\section{Resultados}

A Figura 4 apresenta a ilustração com resultado final da confecção de peça anatômica do olho de baixo custo.

Figura 4. Ilustração final da peça anatômica do olho.
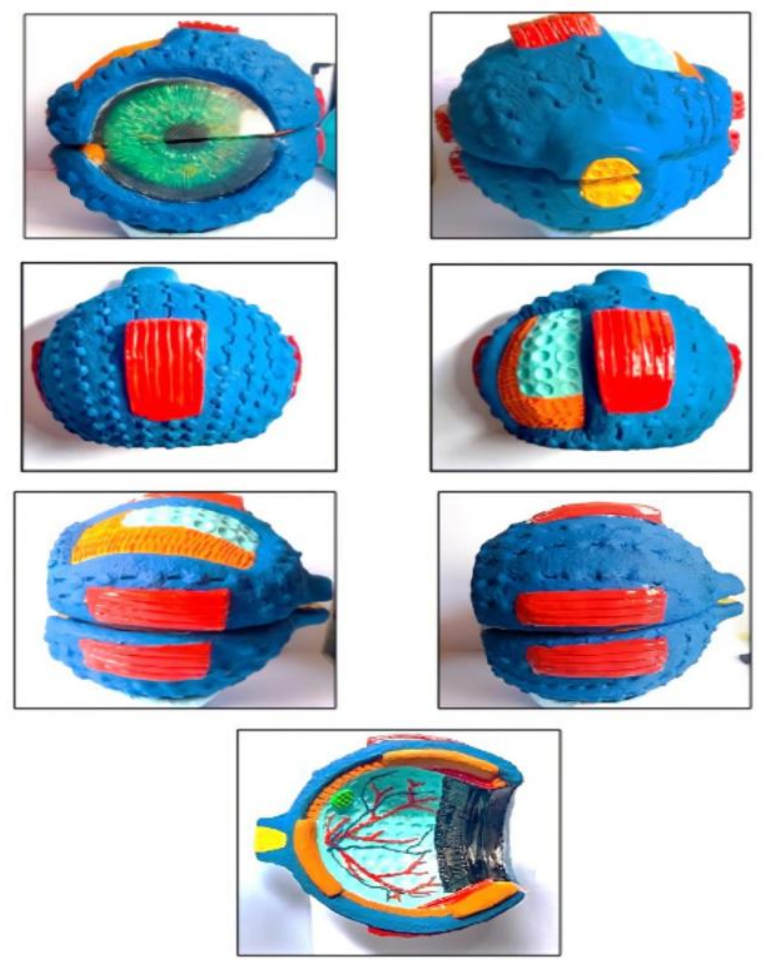

Fonte: Autores.

A peça anatômica construída com materiais de baixo custo consiste em um olho com as estruturas anatômicas geralmente encontradas em atlas de anatomia, como esclera, retina, coróide, córnea, cristalino, íris, musculatura intrínseca (músculos: reto medial, reto lateral e reto inferior), nervo óptico, artérias e veias do órgão, humor vítreo, fóvea e corpo ciliar. O modelo anatômico do olho apresenta na superfície de cada pequena estrutura relevos e textura específica, onde se atentou as diferentes moldagens estruturais para uma sensibilidade tátil diferenciada dos deficientes visuais. Outrossim, é que a utilização de diferentes texturas e relevo possibilita a diferenciação das estruturas com contrastes do tipo: liso/áspero e fino/espesso (Sant'Anna et al., 2014).

\section{Discussão}

Considerando a escolarização de estudantes cegos ou de baixa visão, é importante o uso dos sentidos remanescentes e de recursos didáticos adaptados (Claudia; Pontes, 2018). Dessa maneira, usar a coloração significativa na peça anatômica, é uma das vantagens da mesma, contribuindo para o melhor uso por parte de deficientes visuais com visão subnormal, isto é, com perda parcial do sentido. Sant'Anna et al. (2014), também afirma que as cores utilizadas nos modelos e esquemas são de tons 
preferencialmente fortes e contrastantes chamando a atenção de qualquer pessoa com visão normal e permitindo a estimulação visual do aluno de baixa visão.

A acessibilidade, maleabilidade e o custo influenciaram na escolha dos materiais utilizados na produção das peças anatômicas. Materiais acessíveis, de baixo custo e que não necessitam de ambientes especializados são ideais para melhoria do processo de ensino-aprendizagem, uma vez que possibilitam sua replicação e utilização em contextos com baixa disponibilidade de recursos, possibilitando uma maior democratização do conhecimento (Silva; Leal, 2017; Sartori \& Ferreira, 2020). A maleabilidade dos materiais vai depender da exigência imposta da estrutura que se pretende produzir, levando em consideração aspectos como: secagem rápida, leveza, acabamento transparente, durabilidade e resistência.

Segundo Magalhães e Kawakami (2020), os estudos evidenciam a importância de estratégias de identificação, prevenção e a intervenção pedagógica aos deficientes visuais com o objetivo de reduzir possíveis dificuldades de aprendizagem de tais. Nesse sentido, os educadores sentem dificuldades no processo de inclusão desses grupos, evidenciando um despreparo dos mesmos, entretanto a utilização de material didático inclusivo auxilia de forma eficiente o processo de ensino-aprendizagem estimulando a percepção desses alunos por intermédio dos sentidos remanescentes como o tato (Sant’Anna et al., 2014).

Pensando nessa perspectiva confeccionou-se a legenda com porções das devidas superfícies estruturais do modelo anatômico e identificou-as com o registro em Braille, visto que o Sistema Braille é um recurso essencial na escolarização e inclusão de educandos cegos no sistema de ensino, permitindo à pessoa cega comunicar-se pela escrita individual (Figura 5). $\mathrm{O}$ Braille é um processo de escrita e leitura, baseado em 64 símbolos em relevo, sendo um processo de aprendizado gradativo, sequencial e lento, visto que a leitura é realizada por meio de movimentos táteis (Gonçalves et al., 2016).

Figura 5. Ilustração da legenda com superfícies estruturais distintas e braille das mesmas.

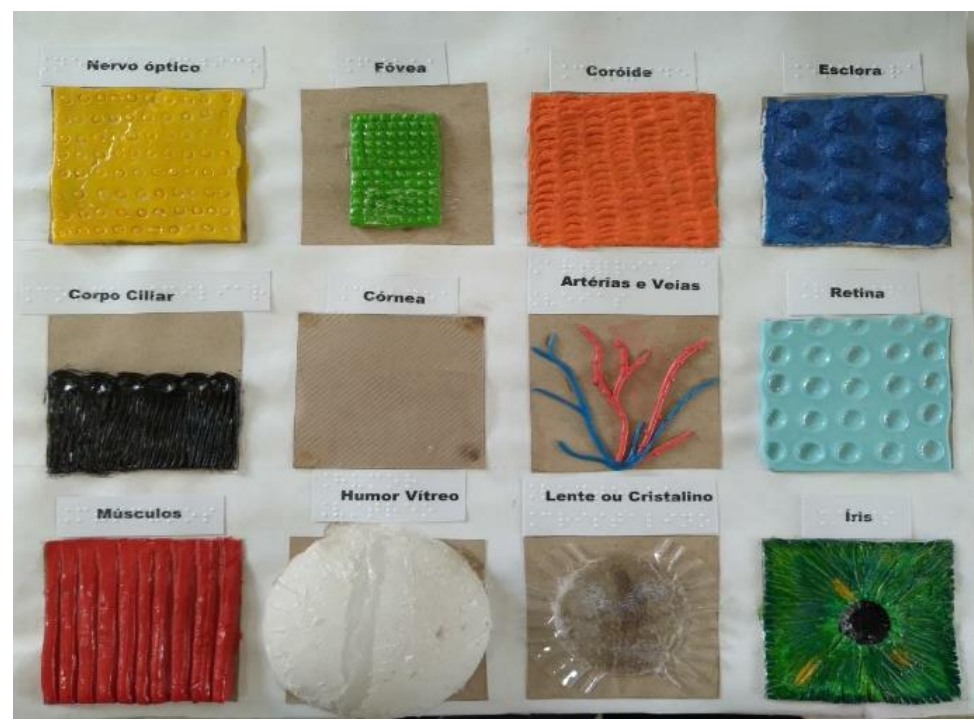

Fonte: Autores.

A deficiência visual priva o indivíduo do mundo das imagens, fazendo-se necessário que outras formas de compreensão sejam estimuladas para o seu desenvolvimento cognitivo, sendo de extrema importância que os estímulos táteis e auditivos para alcançar tal desenvolvimento. Assim sendo, o estímulo tátil se torna imprescindível, tanto no caso da peça anatômica de baixo custo, com a presença de diversos relevos e texturas, como em outras áreas do conhecimento. Se associadas com descrição verbal ou pela leitura do Sistema Braille, torna-se um passo importante para o desenvolvimento pessoal e acadêmico do indivíduo (Dionísio \& Vectore, 2017; Viginheski et al., 2014; Balsaneli \& Treviso, 2015) 
Convém pontuar, ainda, que o deficiente visual enfrenta inúmeros obstáculos em seu processo de inclusão na sociedade, essas dificuldades não se limitam apenas ao acesso à educação, mas em diversos aspectos como a saúde, cultura, mercado de trabalho e informação. Fazendo-se necessário que cada espaço possa se adaptar e garantir a inclusão dessas pessoas. Nesse contexto, com o advento de novas tecnologias e da modernização nos diversos setores, o ambiente acadêmico deve ser palco desse processo de inclusão: os laboratórios de anatomia, bem como as ligas acadêmicas podem ser os pilares desse processo, através de suas atividades de pesquisa, ensino e extensão (Lauter et al., 2011; Silva, 2018; Alves et al., 2019).

\section{Conclusão}

Dado o exposto, conclui-se que o modelo tridimensional acompanhado com a legenda em Braille contendo os nomes das estruturas anatômicas, sendo confeccionado com materiais de baixo custo, apresenta um método alternativo para o ensino aprendizado de educandos com deficiência visual e baixa visão. O modelo ilustrativo do olho abrange em suas estruturas anatômicas formas, texturas e relevos distintos. Dessa forma, o grupo estudado pode criar mentalmente a forma da peça através da percepção tátil, tendo uma aproximação da realidade das estruturas específicas do modelo anatômico. Além disso, por ser um material resistente e leve, proporciona segurança em seu manuseio o que resulta na aprovação da peça, assim como também as cores fortes e o alto contraste em diferentes tonalidades para identificação e compreensão do público alvo que possui baixa visão, o que acarreta na facilidade do processo de ensino.

Futuras pesquisas podem confeccionar peças anatômicas com acessibilidade a deficientes visuais de outros órgãos ou sistemas do corpo humano. Ademais, também é necessário que se pesquise a efetividade das peças anatômicas voltadas a deficientes visuais no processo de ensino-aprendizagem dessa população.

\section{Referências}

Alves, S. P. F., Fernandes, J. G, Braz, A. C. A. R., \& Cardeal, A. L. (2019). Ensino da anatomia humana e inclusão reflexões e possibilidades para a pessoa com

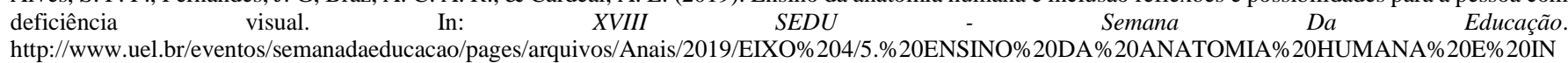
CLUSAO\%20\%20REFLEXOES\%20E\%20POSSIBILIDADES\%20PARA\%20A\%20PESSOA\%20COM\%20DEFICIENCIA\%20VISUAL.pdf

Balsaneli, H. M., \& Treviso, V. C. (2015). Crianças com deficiência visual e o braile. Cadernos de Educação: Ensino e Sociedade, 2(1), 155-168. https://www.unifafibe.com.br/revistasonline/arquivos/cadernodeeducacao/sumario/35/06042015200406.pdf

Brasil (2020). Decreto ${ }^{\circ}$ 10.502, de 30 de setembro de 2020. Institui a Política Nacional de Educação Especial: Equitativa, Inclusiva e com Aprendizado ao Longo da Vida. Diário Oficial da União. 1(189). 6. https://www.in.gov.br/en/web/dou/-/decreto-n-10.502-de-30-de-setembro-de-2020-280529948.

Cerqueira, J. B., \& Ferreira, E. M. B. (2000). Recursos didáticos para a Educação. Revista Benjamin Constat. (156)28. http://www.ibc.gov.br/images/conteudo/revistas/benjamin_constant/2000/edicao-15-abril/Nossos_Meios_RBC_RevAbr2000_ARTIGO3.pdf.

Claudia, A., \& Pontes, N. (2018). O uso de recursos didáticos adaptados na escolarização e inclusão de educandos cegos e de baixa visão. In: IV Colóquio LusoBrasileiro de Educação e II Currículo, Inclusão e Educação Escolar. 1-21. https://periodicos.udesc.br/index.php/colbeduca/article/view/11486.

Costa, A. B., Picharillo, A. D. M., \& Paulino, V. C. (2018). O processo histórico de inserção social da pessoa cega: da Antiguidade à Idade Média. Revista Educação Especial, 31(62), 539. https://periodicos.ufsm.br/educacaoespecial/article/view/24092/pdf.

Dionísio, A. M. P, \& Vectore, C. (2017). Intervenção Mediacional na aprendizagem do Braille: um estudo com crianças deficientes visuais. Psicol. Esc. Educ. 21(3). https://www.scielo.br/j/pee/a/fGxTXtTcwny5dZ5HgcXX6MC/?lang=pt

Fernandes, L. B, Schlesener, A, \& Mosquera, C. (2011). Breve histórico da deficiência e seus paradigmas. Revista do Núcleo de Estudos e Pesquisas Interdisciplinares em Musicoterapia, (2), 132 - 144. http://periodicos.unespar.edu.br/index.php/incantare/article/viewFile/181/186.

Garcez, S. F. (2014). Técnicas para produção e reprodução de material educacional de baixo custo na área de ciências morfológicas para deficientes visuais. InterSciencePlace, (30), 14-32. http://www.interscienceplace.org/isp/index.php/isp/article/view/289. Acesso em: 16 nov. 2020

Gonçalves, H. M., Mourão N. M., \& Engler, R. C. (2016). Design, educação e tecnologias sociais: soluções acessíveis em produtos didático-pedagógicos para o ensino de Braille para cegos. Congresso brasileiro de pesquisa e desenvolvimento em designer. Num.2. vol.9. procediings.blucher.com.br. Acesso em: nov. 2021

Lauter, D. S, Oliveira, D, Fontela, P. C, Cassef, F. D., \& Diel, L. F. (2011). Laboratório de anatomia humana como um espaço de inclusão e vivências: Um relato de experiência. Revista Contexto \& Saúde 10(20), 1065-1070. 
Research, Society and Development, v. 11, n. 2, e54611225555, 2022

(CC BY 4.0) | ISSN 2525-3409 | DOI: http://dx.doi.org/10.33448/rsd-v11i2.25555

Leão, G. B. O. S., \& Sofiato, C. G. (2019). A educação de cegos no Brasil do século XIX: revisitando a história. Revista Brasileira de Educacao Especial, 25(2), 283-300. https://www.scielo.br/scielo.php?script=sci_arttext\&pid=S1413-65382019000200283.

Magalhães, P. G. S., \& Kawakami, L. M. M. (2020). Recursos Didáticos para alunos com Deficiência Visual: Uma análise das Pesquisas no Brasil. Rev. Mult. Psic. 14(50), 1153-1169. https://idonline.emnuvens.com.br/id/article/view/2541/3855.

Nuernberg, A. H. (2008). Contribuições de Vigotski para a educação de pessoas com deficiência visual. Psicologia em Estudo, 13(2), 307-316. https://www.scielo.br/scielo.php?pid=S1413-73722008000200013\&script=sci_abstract\&tlng=pt.

Praça, F. S. G. (2015). Metodologia da pesquisa científica: organização estrutural e os desafios para redigir o trabalho de conclusão. Revista Eletrônica "Diálogos Acadêmicos”. 08(1), 72-87. http://www.uniesp.edu.br/sites/_biblioteca/revistas/20170627112856.pdf

Rogalski, S. M. (2010). Histórico do Surgimento da Educação Especial. Revista de Educação do IDEAU, 5(12), 1-13. https://www.passofundo.ideau.com.br/wpcontent/files_mf/eca97c3f3c5bda644479e4c6a858f556168_1.pdf.

Sant'Anna, N. F, Araújo, G.S.M, Rocha, L.O, Garcez, S.F, \& Barboza, C.B. (2014). Técnicas para produção e reprodução de material educacional de baixo custo na área de ciências morfológicas para deficientes visuais. InterSciencePlace - Revista Científica Internacional. (30)2. http://www.interscienceplace.org/isp/index.php/isp/article/view/289. Acesso em: 29 nov. 2020

Sant’Anna, N. F. (2014). Inclusão de deficientes visuais na universidade. In: Congreso Iberoamericano de Ciencia, Tecnologia, Innovación y Educación, 2014, p.1-12 https://www.oei.es/historico/congreso2014/memoriactei/157.pdf

Sant’Anna, N. S., Araújo, G. S. M, Rocha, O. R., Garcez, S. F. G., \& Barboza, C. B. (2014). Técnicas para produção e reprodução de material educacional de baixo custo na área de ciências morfológicas para deficientes visuais. InterSciencePlace - Revista Científica Internacional. $\mathrm{N}^{\circ} 30$, volume IX, artigo nº 2 . D.O.I: $10.6020 / 1679-9844 / 3002$

Silva, D. S. (2018). Suportes para estudantes com deficiência visual no ensino superior. Dissertação (mestrado) - Universidade Federal de São Carlos, campus São Carlos, São Carlos. https://repositorio.ufscar.br/bitstream/handle/ufscar/10157/SILVA_DANIELESENTEVILDA_2018.pdf?sequence=3\&isAllowed=y

Silva, J. C. X., Leal, C. E. \& Dos S. (2017). Proposta de laboratório de física de baixo custo para escolas da rede pública de ensino médio. Revista Brasileira de Ensino de Física, 39(1), 1401. https://www.scielo.br/j/rbef/a/FrKxnmJjJmYTyptKtnGppjc/abstract/?lang=pt

Viginheski, L. V. M, Frassom, A. C, Silva, S, \& Shimazaki, E. M. (2014). O sistema Braille e o ensino da Matemática para pessoas cegas. Revista Ciência \& Educação. 20(4). http://dx.doi.org/10.1590/1516-73132014000400009 